\title{
Saturation mutagenesis of a +1 programmed frameshift-inducing mRNA sequence derived from a yeast retrotransposon
}

\author{
CARLA GUARRAIA, ${ }^{1}$ LAURA NORRIS, ANA RAMAN, and PHILIP J. FARABAUGH \\ Department of Biological Sciences and Chemistry/Biology Interface Program, University of Maryland Baltimore County, Baltimore, \\ Maryland 21250, USA
}

\begin{abstract}
Errors during the process of translating mRNA information into protein products occur infrequently. Frameshift errors occur less frequently than other types of errors, suggesting that the translational machinery has more robust mechanisms for precluding that kind of error. Despite these mechanisms, mRNA sequences have evolved that increase the frequency up to 10,000-fold. These sequences, termed programmed frameshift sites, usually consist of a heptameric nucleotide sequence, at which the change in frames occurs along with additional sequences that stimulate the efficiency of frameshifting. One such stimulatory site derived from the Ty3 retrotransposon of the yeast Saccharomyces cerevisiae (the Ty3 stimulator) comprises a 14 nucleotide sequence with partial complementarity to a Helix 18 of the $18 \mathrm{~S}$ rRNA, a component of the ribosome's accuracy center. A model for the function of the Ty3 stimulator predicts that it base pairs with Helix 18, reducing the efficiency with which the ribosome rejects erroneous out of frame decoding. We have tested this model by making a saturating set of single-base mutations of the Ty3 stimulator. The phenotypes of these mutations are inconsistent with the Helix 18 base-pairing model. We discuss the phenotypes of these mutations in light of structural data on the path of the mRNA on the ribosome, suggesting that the true target of the Ty3 stimulator may be rRNA and ribosomal protein elements of the ribosomal entry tunnel, as well as unknown constituents of the solvent face of the $40 \mathrm{~S}$ subunit.
\end{abstract}

Keywords: Saccharomyces cerevisiae; programmed frameshifting; ribosome structure

\section{INTRODUCTION}

The ribosome is a large ribonucleoprotein complex that orchestrates the process of protein synthesis from an mRNA template (Green and Noller 1997; Ogle et al. 2003; Wintermeyer et al. 2004; Nilsson and Nissen 2005). The extension of a growing polypeptide chain proceeds by sequential recruitment of aminoacyl-tRNAs (aa-tRNAs) as a ternary complex with elongation factor 1A and GTP (aatRNA $\bullet E F-1 A \bullet G T P$ ) to a binding site on the ribosome, the A site. The ribosome then transfers the growing peptide chain to the aminoacyl-tRNA from a peptidyl- tRNA bound to

${ }^{1}$ Present address: Department of Chemical and Biochemical Engineering, University of Maryland Baltimore County, Baltimore, MD 21250, USA.

Reprint requests to: Philip J. Farabaugh, Department of Biological Sciences and Chemistry/Biology Interface Program, University of Maryland Baltimore County, Baltimore, MD 21250, USA; e-mail: farabaug@ umbc.edu; fax: (410) 455-3875.

Article published online ahead of print. Article and publication date are at http://www.rnajournal.org/cgi/doi/10.1261/rna.735107. the $\mathrm{P}$ site. The translational machinery employs multiple mechanisms to reduce the frequency of errors during protein synthesis, discriminating between correct (cognate) and incorrect (near or noncognate) aa-tRNAs by a process involving both kinetic proofreading (Hopfield 1974; Ninio 1975) and an induced fit mechanism (for review, see Rodnina et al. 2005). Kinetic proofreading can select against incorrect aa-tRNAs because of their rapid dissociation during two successive selection steps before and after GTP hydrolysis by EF-1A (for review, see Thompson 1988). The greatest effect on accuracy, however, appears not to result from these kinetic effects but rather from the ability of cognate aa-tRNAs to induce a change in ribosome structure increasing the rate of EF-1A GTPase activation and thus accelerating their acceptance relative to incorrect aa-tRNAs (for review, see Rodnina et al. 2005).

Despite these mechanisms, translation is not completely accurate partly because of the need to maximize translational output. For example, to achieve an elongation rate of 10 amino acids per second while still allowing correct tRNAs to outcompete the vast excess of incorrect tRNAs, 
the intrinsic rate of GTP hydrolysis must be extremely fast (Gromadski and Rodnina 2004). The reaction is kept far enough from equilibrium that differences in intrinsic stability cannot increase accuracy. Thus, maximizing protein output through GTP activation fails to maximize accuracy (Gromadski and Rodnina 2004).

The error rate is far from constant; it varies significantly with changes in mRNA sequence context (for review, see Parker 1989). The origin of context effects remains largely unexplained. Some variation could result from differences in the interaction between the mRNA and the ribosome's accuracy center. An extreme form of context effect occurs at mRNA sequences, termed "recoding sites" (Gesteland et al. 1992), which induce translational errors at a defined site. Recoding encompasses a variety of phenomena including programmed readthrough of nonsense codons, frameshifting, and bypassing of several codons (Farabaugh 1996; Namy et al. 2004). Errors at recoding sites occur at rates approaching 50\% per codon (Farabaugh 1996; Namy et al. 2004), three orders of magnitude greater than the background rate of $\sim 5 \times 10^{-4}$ per codon (Parker 1989).

Programmed frameshifting occurs typically by shifts of 1 nucleotide (nt) in either the $3^{\prime}$ direction with respect to the mRNA (e.g., programmed -1 frameshifting) or the $5^{\prime}$ direction (e.g., programmed +1 frameshifting). Programmed +1 frameshifting in Saccharomyces cerevisiae occurs at special heptameric sites composed of codons that occupy the ribosomal $\mathrm{P}$ and A sites during the frameshift. Frameshifting requires that during the shift a near-cognate peptidyl-tRNA occupy the P site codon that forms a weak pyrimidine•pyrimidine or a purine•purine third position ("wobble") pair (Sundararajan et al. 1999). We have hypothesized that the unusual pairing in the $\mathrm{P}$ site disturbs the suite of interactions between the codon anticodon complex and the ribosome and interferes with acceptance of even perfect cognate tRNAs in the A site (Stahl et al. 2002). This effect would eliminate the nearly absolute preference for in-frame cognate tRNAs and increase the probability of frameshifting by recruitment of a cognate tRNA in the +1 frame. The ability of the P site tRNA to slip +1 on the mRNA further increases frameshift efficiency (Belcourt and Farabaugh 1990; Sundararajan et al. 1999). The requirement for slippage is not absolute; frameshifting can also occur by out-of-frame recruitment of aa-tRNA without slippage (Farabaugh et al. 1993; Sundararajan et al. 1999).

Sequences surrounding a recoding site can stimulate translational errors (Larsen et al. 1995; Farabaugh 1996; Namy et al. 2004). Some sequences locally reduce the rate of canonical translation, inducing a translational pause. Ribosome pausing may provide sufficient time for a slow noncanonical event (frameshift, readthrough, or bypass). For example, the efficiency of +1 frameshifting in yeast is determined by competition between tRNAs that recognize the zero and +1 frame codons in the A site. Slow recog- nition of an in-frame codon tends to increase frameshifting efficiency (Belcourt and Farabaugh 1990; Kawakami et al. 1993), as does rapid recognition of the overlapping +1 frame codon (Pande et al. 1995). Alternatively, context sequences can directly stimulate the frequency of the noncanonical decoding event. For example, programmed frameshift sites in bacteria include a Shine-Dalgarno (SD) site immediately upstream of the shift site (Larsen et al. 1995). The sequence appears to base pair with the anti-SD site near the $3^{\prime}$ end of the $16 \mathrm{~S}$ rRNA. The spacing between the SD and shift sites is not optimal. The mRNA•rRNA pairing may introduce strain that is lessened by the ribosome moving into the shifted frame. Thus, the SD interaction would effectively pull the ribosome into the shifted frame (Curran and Yarus 1988; Weiss et al. 1988).

The mechanism of other context effects remain unexplained. For example, a 14-nt sequence downstream from the retrotransposon $\mathrm{Ty} 3$ frameshift site in the yeast S. cerevisiae, termed the Ty3 stimulator, increases programmed +1 frameshifting by as much as 7.5 -fold (Farabaugh et al. 1993). It functions as a primary mRNA sequence has, and has limited complementarity with a region of the rRNA within the loop of Helix 18, an element of the ribosome's accuracy center ( $\mathrm{Li}$ et al. 2001). This target region includes a highly conserved nucleotide with a major role in aa-tRNA recognition in the A site, guanosine 577 (G577), which corresponds to G530 of bacterial ribosomes. In bacterial ribosomes, G530 contacts both the second and third base pair of the cognate codon•anticodon complex, facilitating its recognition by the ribosome (for review, see Ogle et al. 2003). DNA cross-linking assays showed that only when the A site is empty, G530 lies very close to the mRNA region corresponding to the Ty3 stimulator (Dontsova et al. 1992; Rinke-Appel et al. 1993). Based on the complementarity and proximity of the mRNA and rRNA, Li et al. (2001) predicted that the Ty3 stimulator might pair with this region (Fig. 1) and interfere with its rapid recognition of

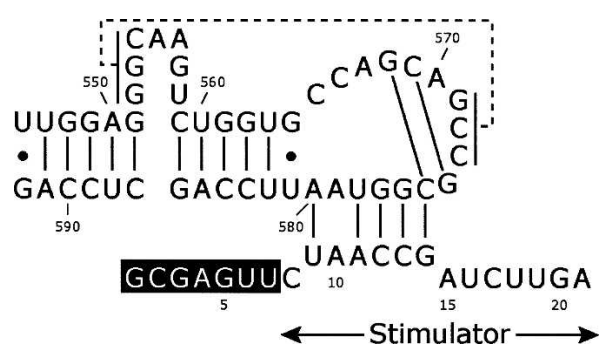

FIGURE 1. The Helix 18 pairing model. The Ty3 stimulator was proposed to pair with the loop of Helix 18 at a short region of complementarity as shown (after Li et al. 2001). Black lines indicate Watson-Crick base pairing; dots indicate $\mathrm{G} \bullet \mathrm{U}$ non-Watson-Crick pairing. The dashed line indicates a pseudoknot tertiary interaction within Helix 18. Base pairing within Helix 18 is based on the most recent predicted secondary structure of Gutell (1996). Note that pairing of C575 to G14 of the stimulator would necessitate disruption of the G568-C575 pair within Helix 18. 
cognate aa-tRNA. The data supporting this conclusion include a set of missense mutations across the Ty3 stimulator. Almost all of these mutations had phenotypes consistent with the model. We have revisited this issue to further test the Helix 18 pairing model by constructing a saturating set of single-base missense mutations. These data show that there is no correlation between the effect of these mutations on frameshift stimulation and on predicted pairing. We conclude that the Helix 18 pairing model is incorrect and suggest an alternative explanation for the Ty3 stimulator effect.

\section{RESULTS AND DISCUSSION}

\section{A saturating set of single-base point mutations of the Ty3 stimulator}

The genetic evidence that provided support for the Helix 18 pairing model (Fig. 1) consisted of a set of 16 single and double-mutations of the Ty3 stimulator, which together altered 13 out of 14 of the stimulator bases (Li et al. 2001). The stimulator corresponds to the nucleotides 8-21 downstream of the $5^{\prime}$ nucleotide of the $\mathrm{P}$ site codon, GCG. Mutations of positions 8-15 tended to reduce frameshift stimulation about twofold, but some had no effect (A10U and A11C); only one mutation (U9C) much more strongly reduced stimulation ( $\mathrm{Li}$ et al. 2001). Eight of the mutations altered positions predicted to base pair with Helix 18, and five of those had effects that were consistent with the model; that is, mutations predicted to weaken the predicted pairing also reduced stimulation. Of the three other relevant mutations, only one (A11C) was explicitly inconsistent with the model, since it failed to reduce stimulation even though it was predicted to replace an $\mathrm{A}-\mathrm{U}$ pair with a pairing of $\mathrm{C}$ and $\mathrm{U}$, which cannot form a Watson-Crick base pair. The combination of genetic results that generally support the model and cross-linking data suggesting that the mRNA and rRNA regions were in close apposition (Dontsova et al. 1992) increased the plausibility of the model, although it remained hypothetical.

A critical test of the model would have involved combining one or more defective mutants of the Ty3 stimulator with mutations altering rRNA nucleotides to which they are predicted to pair. We could conclude that the pairing model were correct if we found that pairing with the mutant stimulator restored frameshifting. This kind of analysis has been successfully used to test many RNA-RNA pairing schemes, for example, testing mRNA•snRNA pairing (Schaufele et al. 1986; Zhuang and Weiner 1986; Parker et al. 1987). Unfortunately, mutations altering the rRNA region predicted to pair with the Ty3 stimulator confer either a lethal or extremely error-prone phenotype (Powers and Noller 1990; Santer et al. 1993; Taliaferro and Farabaugh 2007b). We have therefore chosen to test the model in a different way. The fact that the previous missense mutagenesis created only a small subset of all possible mutations weakens support for the pairing model, because, by chance, the subset tested may fail to invalidate the model, because untested mutations might exist that would have invalidated it. To be sure whether this is the case, we constructed and tested a saturating set of missense mutations of the Ty3 stimulator.

The mutations were constructed in the context of the frameshift reporter plasmid created by $\mathrm{Li}$ et al. (2001), in which the Ty3 frameshift site is fused to a downstream reporter, the bacterial lac $Z$ gene encoding $\beta$-galactosidase. Expression of the enzyme requires the ribosome to enter the frameshift site in the zero frame and shift into the +1 frame and continue translating into lac $Z$. To calculate frameshifting efficiency, the activity of $\beta$-galactosidase from this construct is compared with the activity of a matched reporter, in which lac $Z$ is in the zero frame and therefore does not require frameshifting for its expression. Each of the 42 possible single-base mutations of the 14-nt Ty3 stimulator were constructed, and the activity of $\beta$-galactosidase expressed from each mutant plasmid determined as described in Materials and Methods.

The values we obtained for frameshift stimulation by the Ty3 stimulator mutants C8U, U9C, U9A, A10C, A11C, C13G, G14A, and U18G agree within experimental error with our previous data ( $\mathrm{Li}$ et al. 2001). The sole exception is the mutation $\mathrm{A} 10 \mathrm{U}$, which had been reported not to alter stimulation; we find that it reduces stimulation $\sim 2.5$-fold. This appears to reflect an error in the original data.

Altering stimulator nucleotides proposed under the model to base pair with Helix 18 should have predictable effects. The Helix 18 pairing model proposes five WatsonCrick base pairs: U9•A580, A11•U578, C12•G577, $\mathrm{C} 13 \bullet \mathrm{G} 576$, and $\mathrm{G} 14 \bullet \mathrm{C} 575$. Watson-Crick pairs are isomorphic only with other Watson-Crick pairs, because the distance between the sugar-phosphate backbones of Watson-Crick pairs is different from any non-Watson-Crick pair (Gautheret and Gutell 1997). We would predict that mutating any of these putative Watson-Crick pairs should reduce stimulator activity. Replacing Watson-Crick with non-Watson-Crick pairs in verified RNA $\bullet$ RA helices generally destabilizes pairing. This can be seen most easily in the phylogenetic analysis of rRNA, in which sequence changes within internally paired regions generally restore $\mathrm{A} \bullet \mathrm{U}, \mathrm{G} \bullet \mathrm{C}$, or $\mathrm{G} \bullet \mathrm{U}$ pairing by introducing compensatory changes in the two-paired regions of the molecule (for example, see Hancock et al. 1988). The model also predicts that A10 is opposite A579, implying the possiblity of a nonWatson-Crick A•A pair. As shown in Figure 2, adenines can form non-Watson-Crick base pairs in three ways: WatsonCrick/Watson-Crick (N1:N6 and N6:N1), Watson-Crick/ Hoogsteen (the Watson-Crick pairing groups N1 and N6 pairing with the Hoogsteen pairing groups N6 and N7, respectively), and Hoogsteen/Hoogsteen (N6:N1 and N1: N6). None of these potential non-Watson-Crick pairs are 

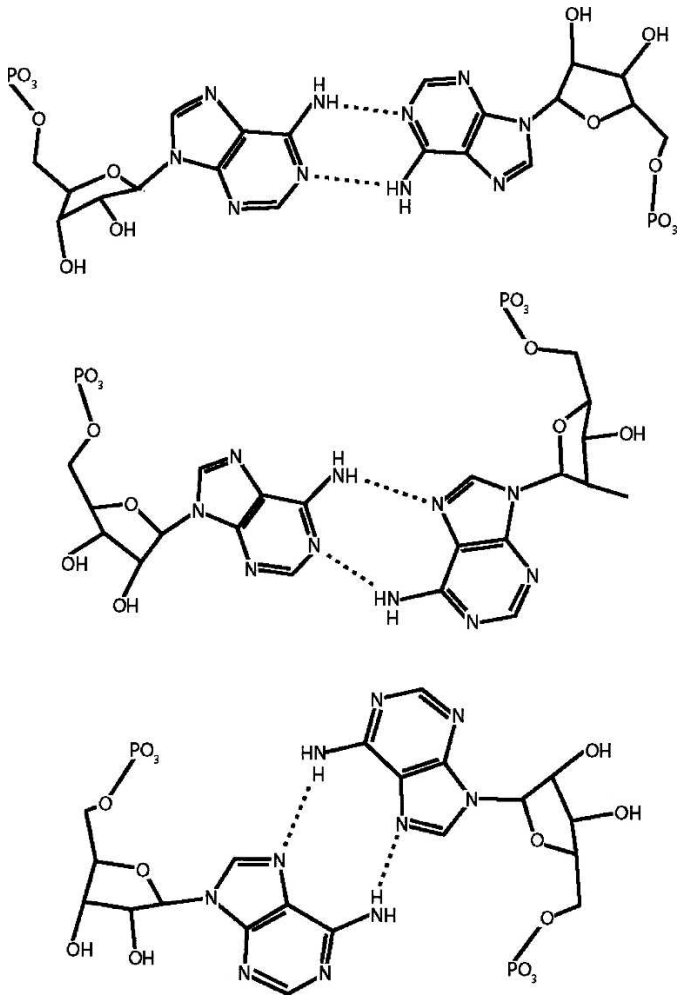

FIGURE 2. Structure of non-Watson-Crick A $\bullet$ A base pairs. WatsonCrick/Watson-Crick (top), Watson-Crick/Hoogsteen (middle), and Hoogsteen/Hoogsteen (bottom). Dotted lines indicated predicted hydrogen bonds. Note the decreasing distance between the phosphodiester backbone of the three pairs and the fact that the WatsonCrick/Hoogsteen pair is parallel rather than antiparallel.

isomorphic (Gautheret and Gutell 1997), so the exact structure of a complex including an $A \bullet A$ pair would depend on the base pair formed. The three $A \bullet A$ pairs, however, are isomorphic with specific $A \bullet G, A \bullet C$, and $G \bullet G$ pairs, respectively (Gautheret and Gutell 1997). If Helix 18 pairing required a specific conformation at A10 we would expect that one, although not both of the mutants A10G or A10C might mimic that conformation and provide wild-type activity. If, on the other hand, the conformation of the A10 pair were not essential, any Watson-Crick $(\mathrm{A} \cdot \mathrm{U})$ or non-WatsonCrick pair might replace A10•A579 and provide activity.

As shown in Figure 3, our data do confirm that region of proposed Helix 18 pairing is important for frameshift stimulation; over half of the mutations reduce frameshift stimulation, with the decrease averaging threefold. The details of the effects of the mutations, however, are not consistent with the model. All mutations of two positions (U9 and G14) reduce stimulation significantly below the wild-type value; at three other positions (A10, A11, and C12) at least one mutation fails to conform to expectations. At two nucleotides predicted to form Watson-Crick pairs (A11 and C12), one or more mutations have activity indistinguishable from wild type, although the model pre- dicts that all would be defective. In the case of the predicted A10•A579 pair, a specific non-Watson-Crick conformation appears not to be required, since both A10C and A10G have normal activity. On the other hand, the data are inconsistent with the lack of a required conformation, since A10U, which would allow formation of an $A \bullet U$ pair, has reduced activity. We conclude that stimulator activity shows a variable requirement for these six nucleotides, but that the data fail to support the Helix 18 pairing model.

The data show that a $5^{\prime}$ domain (C8-A15) of the stimulator is more critical to stimulation than a $3^{\prime}$ domain (U16-A21). In the $5^{\prime}$ domain, at least one mutation at each position significantly reduced stimulator activity. A total of $79 \%$ (19 of 24) of the $5^{\prime}$ domain mutations reduced stimulation an average of 2.6 -fold. In the $3^{\prime}$ domain, a minority of mutations (6 out of 17 , or $35 \%$ ) reduced activity and only two reduce it more than twofold. One mutation, G20U, creates a +1 frame UAA nonsense codon, blocking expression of the lac $Z$ reporter. The effect of this mutation on stimulation can therefore not be determined. The overall impression of the data is consistent with the conclusions of our previous work that the $5^{\prime}$ domain makes a greater contribution to stimulator activity (Li et al. 2001).

\section{The Ty3 stimulator is located at the mRNA entry channel of the ribosome}

The mRNA bound to bacterial ribosomes is well ordered in high-resolution structures only in the decoding center and for two nucleotides in the $5^{\prime}$ direction (Schuwirth et al.

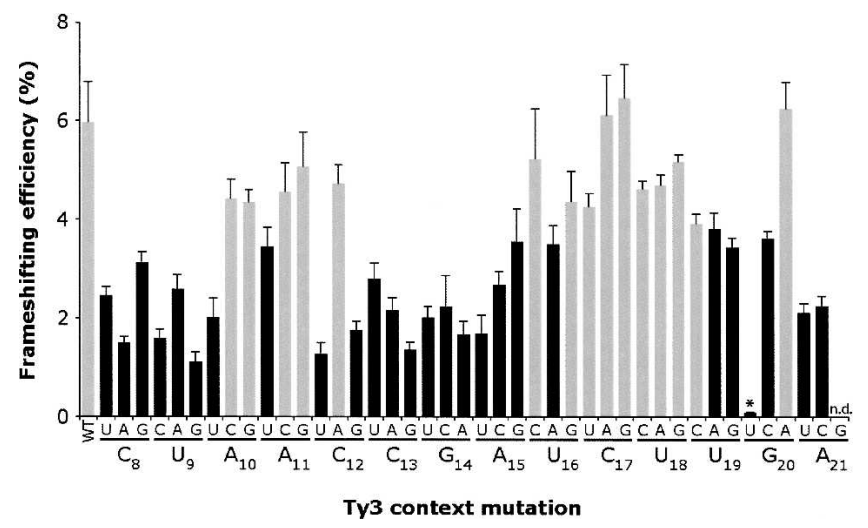

FIGURE 3. Phenotype of mutants of the Ty3 stimulator in exponentially growing cells. Each bar represents $\beta$-galactosidase activity ( \pm SEM) expressed from a reporter involving a wild-type (WT) or missense mutant Ty3 stimulator. Each wild-type nucleotide appears below the graph with a subscript indicating the position; the identity of each mutant tested appears just above in groups of three. Black bars represent results judged by ANOVA as different from wild type $(P \leq$ $0.05)$. The asterisk indicates a mutation (G20U) that introduces an inframe nonsense codon, which strongly reduces expression of lacZ. A21G, which was not included in this set, previously was shown to stimulate $60 \%$ the activity of wild type (Li et al. 2001), a significant reduction in activity. 
2005; Selmer et al. 2006). Yusupova et al. (2001, 2006) mapped the mRNA path, showing that downstream of the decoding center the mRNA enters the ribosome through protein and rRNA layers of a $20 \AA$ diameter entrance tunnel (Fig. 4). The first four nucleotides downstream from the A site (positions 8-11) are within an rRNA layer of the tunnel made up of parts of Helix 2 (the "central pseudoknot"), 18, 28, and 34 (Yusupova et al. 2001). The next five nucleotides (positions 11-15) pass through a second layer, mostly composed of basic residues from three ribosomal proteins, rpS3, rpS4, and rpS5 (Yusupova et al. 2001). These residues constitute the ribosomal helicase responsible for unwinding mRNA secondary structures in advance of the ribosome (Takyar et al. 2005). These three ribosomal proteins, however, also play a role in ribosomal accuracy; ram mutations (ribosomal ambiguity) affecting rpS4 and rpS5 (Birge and Kurland 1970; Deusser et al. 1970; Stoffler et al. 1971) reduced the stringency of decoding (Thompson et al. 1981; Andersson and Kurland 1983) and a mutation in the rpS3 homolog in S. cerevisiae increased suppression of frameshift mutations (Hendrick et al. 2001). Past position 15, the mRNA resides outside of the entrance tunnel, but was too disordered to be localized (Yusupova et al. 2001).

Although these data derive from studies of bacterial ribosomes, comparison of high-resolution X-ray crystallo-

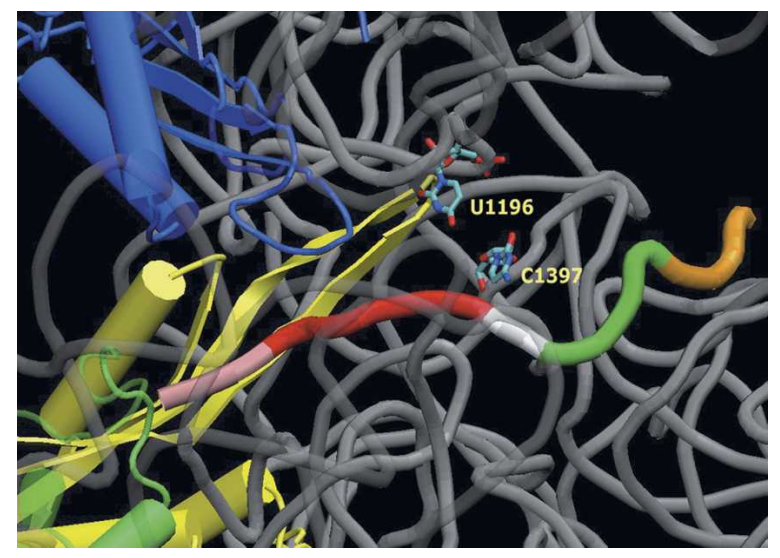

FIGURE 4. Pathway of the mRNA through the ribosome's entrance channel. The figure shows a rendering based on the X-ray structure of the Thermus thermophilus 70 S ribosome in the "post-initiation" state (Yusupova et al. 2006). The multicolored tube represents the mRNA from the P site to position 13. The colors represent the P site codon (orange), A site codon (green), position 7 (white), positions 8-11 (red) corresponding to the rRNA layer of the entrance tunnel, and positions 12-13 (pink) corresponding to the first two nucleotides of the ribosomal protein layer. The gray tubes represent the $16 \mathrm{~S}$ rRNA; the two nucleotides proposed to interact with the mRNA at positions 7 and 9, C1397 and U1196 appear in the center of the image. Ribosomal proteins S3 (blue), S4 (green), and S5 (yellow) are shown in cartoon format at left. Residues in front of the entrance tunnel have been made partially transparent in the image for clarity. The coordinate file used to construct the figure, 2HGP, was obtained from the Protein Data Bank; the image was created using VMD 1.8.6 (Humphrey et al. 1996). graphic structures of bacterial 70S ribosomes (Schuwirth et al. 2005; Selmer et al. 2006) and a lower-resolution cryoelectron microscopy structure of a eukaryotic $80 \mathrm{~S}$ ribosome (Spahn et al. 2001, 2004) demonstrates large-scale similarity in structure and function. The central structures of these ribosomes appear to be conserved. Differences are confined to regions distant from the decoding center. In particular, bacterial and eukaryotic ribosomes each include an entrance tunnel apparently composed of homologous structures.

Comparison of our missense mutations and the predicted path of the mRNA demonstrates that the 5' (C8A15) and 3' (U16-A21) domains of the Ty3 stimulator described above correspond to the regions within and outside of the entrance tunnel. The $5^{\prime}$ domain is divided between nucleotides predicted to be within the rRNA (C8$\mathrm{A} 11)$ or the ribosomal protein layer (C12-A15). At least one mutation at each position of the $\mathrm{C} 8-\mathrm{A} 11$ region strongly reduces stimulation with the greater effect at $\mathrm{C} 8$ and U9. Yusupova et al. (2001) identified a possible interaction between two rRNA nucleotides that point into the entrance channel, C1397 and U1196, and the mRNA at positions 7 and 9, respectively. They propose that these interactions might position the mRNA downstream of the A-site codon (Yusupova et al. 2001). We have not modified U7 of the Ty3 site in these studies, since this nucleotide forms part of the frameshift heptamer and therefore affects frameshifting directly. It is attractive to suggest, however, that $5^{\prime}$ domain mutations might disrupt interactions with C1397 and U1196, which would implicate them in reading frame maintenance. If these nucleotides interact with the region immediately past the A site, their normal function could be to block reading out of frame. Mutations in the region of C1397 (O'Connor et al. 1995, 1997) and U1196 (Goringer et al. 1991; Moine and Dahlberg 1994; O’Connor et al. 1995, 1997) are known to affect translational accuracy.

The downstream half of the $5^{\prime}$ domain $(\mathrm{C} 12-\mathrm{A} 15)$ is predicted to interact with $\mathrm{rpS} 3, \mathrm{rpS} 4$, and $\mathrm{rpS5}$. Our data imply that the residues of these proteins that constitute the ribosomal helicase (Takyar et al. 2005) may have a second role in maintaining translational reading frame. We find that nearly all mutations of $\mathrm{C} 12$ to A15 strongly reduce frameshift stimulation. It is attractive to suggest that the nucleotide sequence in this region ( $5^{\prime}$-CCGA-3') interferes with a function of the ribosomal protein residues in preventing +1 frameshifting. Although genetic evidence already identifies these three proteins in translational accuracy, available mutations do not implicate the helicase center directly in that process. The ram mutations, however, were selected to enhance missense errors and compensate for hyperaccurate mutations of $\mathrm{rpS12}$, the product of the strA gene (Gorini 1974). It may be that mutations in the helicase center do not affect this phenotype, but only affect frame maintenance. We are testing these predictions by directed mutagenesis of the helicase center encoded in the $r p s C$ (rpS3), rpsD (rpS4), and $r p s E$ (rpS5) genes. 
Most mutations to the $3^{\prime}$ domain of the Ty 3 stimulator (U16-A21) have no significant effect on stimulation. The exceptional mutations that cause a significant decrease include U16A and mutations to the $3^{\prime}$ end of the stimulator, U19, G20, and A21. Most of these mutations reduce stimulation less than twofold. The fact that a portion of the $3^{\prime}$ domain does appear to affect stimulation suggests that the Ty3 stimulator interacts with target(s) outside of the ribosome's entrance tunnel, which could include elements of the solvent face of the $40 \mathrm{~S}$ subunit or some nonribosomal factor(s). The region is unlikely to interact directly with either elongation factor (eEF-1A or eEF2); cryoelectron microscopic analysis of EF2 bound to $80 \mathrm{~S}$ ribosomes (Gomez-Lorenzo et al. 2000) and of the eEF-1A homo$\log \mathrm{EF}-\mathrm{Tu}$ bound to $70 \mathrm{~S}$ bacterial ribosomes (Stark et al. 1997) show that these factors bind at a site at the interface between the $40 \mathrm{~S}$ and $60 \mathrm{~S}$ subunits far from the solvent side of the entrance tunnel.

\section{Comparison of two frameshift stimulators suggests conserved interactions with the ribosome}

We recently characterized a stimulator sequence immediately downstream of the programmed +1 frameshift site in the $S$. cerevisiae EST3 gene that is almost twice the length of the Ty3 stimulator, with which it shares no primary sequence similarity (Taliaferro and Farabaugh 2007a). The EST3 stimulator, like the Ty3 stimulator, increases frameshifting eightfold. Progressive 3' deletions of the EST3 stimulator suggest that it comprises three separable domains, each responsible for about twofold stimulation. The downstream boundaries of these domains are at positions 13, 25, and 34, numbered from the P site codon (Taliaferro and Farabaugh 2007a). The data of Yusupova et al. (2001) suggest that the first of these domains is mainly in the rRNA layer of the entrance tunnel and the second and third are in the space beyond the entrance tunnel. Saturation mutagenesis of the EST3 stimulator showed that 35 of 86 mutations tested significantly reduced stimulation relative to wild type, and in one it was significantly increased. These mutations are scattered, but only 11 were predicted to lie in the ribosomal entry tunnel (positions 8-15). The strongest clustering of defective mutations were between position 18 and 29, all predicted to lie outside of the tunnel.

The two stimulators may be more similar than this summary implies. Like the Ty3 stimulator, mutations to positions 8 and 9 , immediately downstream from the frameshift heptamer, generally strongly reduce frameshifting (Taliaferro and Farabaugh 2007a). It may be that these two sequences interfere with frame maintenance by similar mechanisms. The region of the EST3 stimulator predicted to occupy the rpS3/rpS4/rpS5 layer of the entrance channel, corresponding to position 11-17, is a run of pyrimidines, $5^{\prime}$-UUUUCCC-3'. This region is poorly conserved in budding yeast EST3 genes (Taliaferro and Farabaugh 2007a), suggesting that it may not be essential to function. We do not, however, know how these homologous stimulator sequences may affect frameshifting. We do know that some of the missense mutations in this region of the S. cerevisiae sequence do significantly reduce stimulation, which suggests that a functional interaction does exist between the EST3 stimulator and $\mathrm{rpS3} / \mathrm{rpS} 4 / \mathrm{rpS} 5$. The rest of the mutations that reduce EST3 stimulation would fall in the solvent-accessible region past nucleotide 15. A very significant cluster of defective mutants falls within the region from A18 to T30. This region partly overlaps with the 3' domain of the Ty3 stimulator, suggesting that the two may also share a target on the ribosome, though they share little primary sequence similarity. The fact that so many mutations in this region have no significant effect on stimulation suggests that the sequence constraints on the function of the region may be weak and that multiple unrelated sequences might be able to function equivalently.

The analyses of these two frameshift stimulators suggest that the interaction between these mRNA elements and the ribosome may largely involve other than Watson-Crick base pairing. A prejudice in favor of base pairing no doubt derives from the example of the function of base pairing of the Shine-Dalgarno site during translational initiation (Shine and Dalgarno 1974). Although Watson-Crick pairing has been assumed in these interactions because it is fully compatible with the A form of the RNA・RNA helix, the assumption has been questioned more recently by many RNA duplex structures showing involvement of non-Watson-Crick pairs (for review, see Leontis and Westhof 1998; Westhof and Fritsch 2000). Previously, we proposed such an interation to explain the Ty3 stimulator effect (Li et al. 2001). Many others proposed an mRNA•rRNA pairing scheme to explain regulatory mRNA effects (Olins and Rangwala 1989; Thanaraj and Pandit 1989; Ivanov et al. 1995; Resch et al. 1996; Tranque et al. 1998). The validity of these base-pairing interactions has not always been tested explicitly and some have been disproven (for example, the downstream box, O'Connor et al. 1999; La Teana et al. 2000; Moll et al. 2001). It may be that non-base-pairing interactions are much more typical of the interaction of the ribosome with affector RNAs.

\section{MATERIALS AND METHODS}

\section{Bacterial and yeast strains}

The strains used in this work were the Escherichia coli strain DH5 $\alpha$

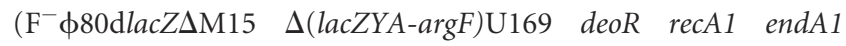
hsdR17( $\mathrm{rk}^{-} \mathrm{mk}^{+}$) phoA supE44 $\lambda^{-}$thi-1 gyrA96 relA1) (Hanahan 1983 ) and S. cerevisiae strain FY1679-18B (Mato, his3-4200, leu2-

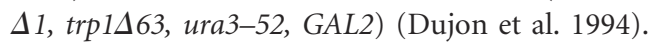

\section{Media and culture conditions}

All E. coli strains were grown in LB medium: $10 \mathrm{~g}$ of Bacto-tryptone (Difco), $5 \mathrm{~g}$ of Bacto yeast extract (Difco), $10 \mathrm{~g}$ of $\mathrm{NaCl}$, and either 
$100 \mu \mathrm{g} / \mathrm{mL}$ ampicillin or $30 \mu \mathrm{g} / \mathrm{mL}$ chloramphenicol. All bacterial transformations were performed using the Z-competent protocol (Zymo research). S. cerevisiae strains were grown on synthetic medium with $2 \%$ glucose (SD) supplemented with amino acids, lacking uracil for plasmid selection. All S. cerevisiae transformations were performed using the LiAc method (Ito et al. 1983).

\section{Plasmid constructions and reporter assay}

Plasmids for the study of Ty3 frameshifting are derivatives of the shuttle plasmid pMB38, a lac Z reporter plasmid that includes the bla gene of pBR322 conferring resistance to ampicillin in bacteria and the URA3 gene of $S$. cerevisiae, a selectable marker for yeast transformation (Belcourt and Farabaugh 1990). Expression of lacZ depends on the transcription and translation from the HIS4 gene, the first 33 codons of which have been fused to lacZ. Saturation mutagenesis was performed using Quickchange XL II protocol in $\mathrm{DH} 5 \alpha$ as recommended by the manufacturer (Stratagene). All DNA oligonucleotides were purchased from Integrated DNA Technologies. Assays of $\beta$-galactosidase in $S$. cerevisiae were essentially as described (Farabaugh et al. 1989). Triplicate assays were conducted on at least three independent transformants. The statistical significance of the data was calculated using the students T-test with data considered significant at the 95\% level.

\section{ACKNOWLEDGMENTS}

This work was supported by a grant to P.J.F. from the National Institutes of Health, R01 GM029480, and in part by the Center for Cancer Research of the National Cancer Institute, Department of Health and Human Services.

Received July 17, 2007; accepted August 8, 2007.

\section{REFERENCES}

Andersson, D.I. and Kurland, C.G. 1983. Ram ribosomes are defective proofreaders. Mol. Gen. Genet. 191: 378-381.

Belcourt, M.F. and Farabaugh, P.J. 1990. Ribosomal frameshifting in the yeast retrotransposon Ty: tRNAs induce slippage on a 7 nucleotide minimal site. Cell 62: 339-352.

Birge, E.A. and Kurland, C.G. 1970. Reversion of a streptomycindependent strain of Escherichia coli. Mol. Gen. Genet. 109: 356-369.

Curran, J. and Yarus, M. 1988. Use of tRNA suppressors to probe regulation of Escherichia coli release factor 2. J. Mol. Biol. 203: 75-83.

Deusser, E., Stoffler, G., and Wittmann, H.G. 1970. Ribosomal proteins. XVI. Altered S4 proteins in Escherichia coli revertants from streptomycin dependence to independence. Mol. Gen. Genet. 109: 298-302.

Dontsova, O., Dokudovskaya, S., Kopylov, A., Bogdanov, A., RinkeAppel, J., Junke, N., and Brimacombe, R. 1992. Three widely separated positions in the 16S RNA lie in or close to the ribosomal decoding region: A site-directed cross-linking study with mRNA analogues. EMBO J. 11: 3105-3116.

Dujon, B., Alexandraki, D., Andre, B., Ansorge, W., Baladron, V., Ballesta, J.P., Banrevi, A., Bolle, P.A., Bolotin-Fukuhara, M., Bossier, P., et al. 1994. Complete DNA sequence of yeast chromosome XI. Nature 369: 371-378.

Farabaugh, P., Liao, X.-B., Belcourt, M., Zhao, H., Kapakos, J., and Clare, J. 1989. Enhancer and silencerlike sites within the transcribed portion of a Ty2 transposable element of Saccharomyces cerevisiae. Mol. Cell. Biol. 9: 4824-4834.

Farabaugh, P.J. 1996. Programmed translational frameshifting. Microbiol. Rev. 60: 103-134.
Farabaugh, P.J., Zhao, H., and Vimaladithan, A. 1993. A novel programed frameshift expresses the POL3 gene of retrotransposon Ty3 of yeast: Frameshifting without tRNA slippage. Cell 74: 93-103.

Gautheret, D. and Gutell, R.R. 1997. Inferring the conformation of RNA base pairs and triples from patterns of sequence variation. Nucleic Acids Res. 25: 1559-1564. doi: 10.1093/nar/25.8.1559.

Gesteland, R., Weiss, R., and Atkins, J. 1992. Recoding: Reprogrammed genetic decoding. Science 257: 1640-1641.

Gomez-Lorenzo, M.G., Spahn, C.M., Agrawal, R.K., Grassucci, R.A., Penczek, P., Chakraburtty, K., Ballesta, J.P., Lavandera, J.L., GarciaBustos, J.F., and Frank, J. 2000. Three-dimensional cryo-electron microscopy localization of EF2 in the Saccharomyces cerevisiae $80 \mathrm{~S}$ ribosome at 17.5 A resolution. EMBO J. 19: 2710-2718.

Goringer, H.U., Hijazi, K.A., Murgola, E.J., and Dahlberg, A.E. 1991. Mutations in 16S rRNA that affect UGA (stop codon)-directed translation termination. Proc. Natl. Acad. Sci. 88: 6603-6607.

Gorini, L. 1974. Streptomycin and misreading of the genetic code. In Ribosomes. (eds. M. Nomura, et al.), pp. 791-803. Cold Spring Harbor Laboratory Press, Cold Spring Harbor, N.Y.

Green, R. and Noller, H.F. 1997. Ribosomes and translation. Annu. Rev. Biochem. 66: 679-716.

Gromadski, K.B. and Rodnina, M.V. 2004. Kinetic determinants of high-fidelity tRNA discrimination on the ribosome. Mol. Cell 13: 191-200.

Gutell, R.R. 1996. Comparative sequence analysis and the structure of $16 \mathrm{~S}$ and $23 \mathrm{~S}$ rRNA. In Structure, evolution, processing and function in protein biosynthesis. (eds. A.E. Dahlberg and R.A. Zimmermann), pp. 111-128. Ribosomal RNA, CRC Press, Boca Raton, FL.

Hanahan, D. 1983. Studies on transformation of Escherichia coli with plasmids. J. Mol. Biol. 166: 557-580.

Hancock, J.M., Tautz, D., and Dover, G.A. 1988. Evolution of the secondary structures and compensatory mutations of the ribosomal RNAs of Drosophila melanogaster. Mol. Biol. Evol. 5: 393-414.

Hendrick, J.L., Wilson, P.G., Edelman, I.I., Sandbaken, M.G., Ursic, D., and Culbertson, M.R. 2001. Yeast frameshift suppressor mutations in the genes coding for transcription factor Mbflp and ribosomal protein S3: Evidence for autoregulation of S3 synthesis. Genetics 157: 1141-1158.

Hopfield, J. 1974. Kinetic proofreading: A new mechanism for reducing errors in biosynthetic processes requiring high specificity. Proc. Natl. Acad. Sci. 71: 4135-4139.

Humphrey, W., Dalke, A., and Schulten, K. 1996. VMD: Visual molecular dynamics. J. Mol. Graph. 14: 33-38.

Ito, H., Fukuda, Y., Murata, K., and Kimura, A. 1983. Transformation of intact yeast cells treated with alkali cations. J. Bacteriol. 153: $163-168$.

Ivanov, I.G., Alexandrova, R.A., Dragulev, B.P., and AbouHaidar, M.G. 1995. A second putative mRNA binding site on the Escherichia coli ribosome. Gene 160: 75-79.

Kawakami, K., Pande, S., Faiola, B., Moore, D.P., Boeke, J.D., Farabaugh, P.J., Strathern, J.N., Nakamura, Y., and Garfinkel, D.J. 1993. A rare tRNA-Arg(CCU) that regulates Tyl element ribosomal frameshifting is essential for Tyl retrotransposition in Saccharomyces cerevisiae. Genetics 135: 309-320.

La Teana, A., Brandi, A., O'Connor, M., Freddi, S., and Pon, C.L. 2000. Translation during cold adaptation does not involve mRNA-rRNA base pairing through the downstream box. RNA 6: 1393-1402.

Larsen, B., Peden, J., Matsufuji, S., Matsufuji, T., Brady, K., Maldonado, R., Wills, N.M., Fayet, O., Atkins, J.F., and Gesteland, R.F. 1995. Upstream stimulators for recoding. Biochem. Cell Biol. 73: 1123-1129.

Leontis, N.B. and Westhof, E. 1998. Conserved geometrical basepairing patterns in RNA. Q. Rev. Biophys. 31: 399-455.

Li, Z., Stahl, G., and Farabaugh, P.J. 2001. Programmed +1 frameshifting stimulated by complementarity between a downstream mRNA sequence and an error correcting region of rRNA. RNA 7: 275-284.

Moine, H. and Dahlberg, A.E. 1994. Mutations in helix 34 of Escherichia coli $16 \mathrm{~S}$ ribosomal RNA have multiple effects on ribosome function and synthesis. J. Mol. Biol. 243: 402-412. 
Moll, I., Huber, M., Grill, S., Sairafi, P., Mueller, F., Brimacombe, R., Londei, P., and Blasi, U. 2001. Evidence against an Interaction between the mRNA downstream box and $16 \mathrm{~S}$ rRNA in translation initiation. J. Bacteriol. 183: 3499-3505.

Namy, O., Rousset, J.P., Napthine, S., and Brierley, I. 2004. Reprogrammed genetic decoding in cellular gene expression. Mol. Cell 13: $157-168$.

Nilsson, J. and Nissen, P. 2005. Elongation factors on the ribosome. Curr. Opin. Struct. Biol. 15: 349-354.

Ninio, J. 1975. Kinetic amplification of enzyme discrimination. Biochimie 57: 587-595.

O'Connor, M., Brunelli, C.A., Firpo, M.A., Gregory, S.T., Lieberman, K.R., Lodmell, J.S., Moine, H., Van Ryk, D.I., and Dahlberg, A.E. 1995. Genetic probes of ribosomal RNA function. Biochem. Cell Biol. 73: 859-868.

O'Connor, M., Thomas, C.L., Zimmermann, R.A., and Dahlberg, A.E. 1997. Decoding fidelity at the ribosomal A and P sites: Influence of mutations in three different regions of the decoding domain in $16 \mathrm{~S}$ rRNA. Nucleic Acids Res. 25: 1185-1193. doi: 10.1093/nar/ 25.6.1185.

O'Connor, M., Asai, T., Squires, C.L., and Dahlberg, A.E. 1999. Enhancement of translation by the downstream box does not involve base pairing of mRNA with the penultimate stem sequence of 16S rRNA. Proc. Natl. Acad. Sci. 96: 8973-8978.

Ogle, J.M., Carter, A.P., and Ramakrishnan, V. 2003. Insights into the decoding mechanism from recent ribosome structures. Trends Biochem. Sci. 28: 259-266.

Olins, P.O. and Rangwala, S.H. 1989. A novel sequence element derived from bacteriophage T7 mRNA acts as an enhancer of translation of the lacZ gene in Escherichia coli. J. Biol. Chem. 264: 16973-16976.

Pande, S., Vimaladithan, A., Zhao, H., and Farabaugh, P.J. 1995. Pulling the ribosome out of frame +1 at a programmed frameshift site by cognate binding of aminoacyl-tRNA. Mol. Cell. Biol. 15: 298-304.

Parker, J. 1989. Errors and alternatives in reading the universal genetic code. Microbiol. Rev. 53: 273-298.

Parker, R., Siliciano, P.G., and Guthrie, C. 1987. Recognition of the TACTAAC box during mRNA splicing in yeast involves base pairing to the U2-like snRNA. Cell 49: 229-239.

Powers, T. and Noller, H.F. 1990. Dominant lethal mutations in a conserved loop in 16S rRNA. Proc. Natl. Acad. Sci. 87: 1042-1046.

Resch, A., Tedin, K., Grundling, A., Mundlein, A., and Blasi, U. 1996. Downstream boxanti-downstream box interactions are dispensable for translation initiation of leaderless mRNAs. EMBO J. 15: $4740-4748$.

Rinke-Appel, J., Junke, N., Brimacombe, R., Dukudovskaya, S., Dontsova, O., and Bogdanov, A. 1993. Site-directed cross-linking of mRNA analogues to $16 \mathrm{~S}$ ribosomal RNA; a complete scan of cross-links from all positions between " +1 " and " +16 " on the mRNA, downstream from the decoding site. Nucleic Acids Res. 21: 2853-2859. doi: 10.1093/nar/21.12.2853.

Rodnina, M.V., Gromadski, K.B., Kothe, U., and Wieden, H.J. 2005. Recognition and selection of tRNA in translation. FEBS Lett. 579: 938-942.

Santer, U., Cekleniak, J., and Santer, M. 1993. The 530 loop of $16 \mathrm{~S}$ ribosomal RNA: Each base change confers its own phenotype. Mol. Biol. Cell 4: 420a.

Schaufele, F., Gilmartin, G.M., Bannwarth, W., and Birnstiel, M.L. 1986. Compensatory mutations suggest that base-pairing with a small nuclear RNA is required to form the $3^{\prime}$ end of $\mathrm{H} 3$ messenger RNA. Nature 323: 777-781.

Schuwirth, B.S., Borovinskaya, M.A., Hau, C.W., Zhang, W., VilaSanjurjo, A., Holton, J.M., and Cate, J.H. 2005. Structures of the bacterial ribosome at 3.5 A resolution. Science 310: 827-834.

Selmer, M., Dunham, C.M., Murphy, F.V.T., Weixlbaumer, A., Petry, S., Kelley, A.C., Weir, J.R., and Ramakrishnan, V. 2006. Structure of the 70S ribosome complexed with mRNA and tRNA. Science 313: 1935-1942.
Shine, J. and Dalgarno, L. 1974. The 3'-terminal sequence of Escherichia coli 16 S ribosomal RNA: Complementarity to nonsense triplets and ribosome binding sites. Proc. Natl. Acad. Sci. 71: 1342-1346.

Spahn, C.M., Beckmann, R., Eswar, N., Penczek, P.A., Sali, A., Blobel, G., and Frank, J. 2001. Structure of the 80 S ribosome from Saccharomyces cerevisiae-tRNA-ribosome and subunitsubunit interactions. Cell 107: 373-386.

Spahn, C.M., Gomez-Lorenzo, M.G., Grassucci, R.A., Jorgensen, R., Andersen, G.R., Beckmann, R., Penczek, P.A., Ballesta, J.P., and Frank, J. 2004. Domain movements of elongation factor eEF2 and the eukaryotic $80 \mathrm{~S}$ ribosome facilitate tRNA translocation. EMBO J. 23: 1008-1019.

Stahl, G., McCarty, G.P., and Farabaugh, P.J. 2002. Ribosome structure: Revisiting the connection between translational accuracy and unconventional decoding. Trends Biochem. Sci. 27: 178-183.

Stark, H., Rodnina, M.V., Rinke-Appel, J., Brimacombe, R., Wintermeyer, W., and van Heel, M. 1997. Visualization of elongation factor $\mathrm{Tu}$ on the Escherichia coli ribosome. Nature 389: 403-406.

Stoffler, G., Deusser, E., Wittmann, H.G., and Apirion, D. 1971. Ribosomal proteins. XIX. Altered S5 ribosomal protein in an Escherichia coli revertant from streptomycin dependence to independence. Mol. Gen. Genet. 111: 334-341.

Sundararajan, A., Michaud, W.A., Qian, Q., Stahl, G., and Farabaugh, P.J. 1999. Nearcognate peptidyl-tRNAs promote +1 programmed translational frameshifting in yeast. Mol. Cell 4: $1005-1015$.

Takyar, S., Hickerson, R.P., and Noller, H.F. 2005. mRNA helicase activity of the ribosome. Cell 120: 49-58.

Taliaferro, D. and Farabaugh, P.J. 2007a. An mRNA sequence derived from the yeast EST3 gene stimulates programmed +1 translational frameshifting. RNA 13: 606-613.

Taliaferro, D. and Farabaugh, P.J. 2007b. Testing constraints on rRNA bases that make nonsequence-specific contacts with the codon•anticodon complex in the ribosomal A site. RNA 13: 12791286.

Thanaraj, T.A. and Pandit, M.W. 1989. An additional ribosomebinding site on mRNA of highly expressed genes and a bifunctional site on the colicin fragment of 16S rRNA from Escherichia coli: Important determinants of the efficiency of translationinitiation. Nucleic Acids Res. 17: 2973-2985.

Thompson, R. 1988. EFTu provides an internal kinetic standard for translational accuracy. Trends Biochem. Sci. 13: 91-93.

Thompson, R.C., Dix, D.B., Gerson, R.B., and Karim, A.M. 1981. Effect of $\mathrm{Mg}^{2+}$ concentration, polyamines, streptomycin, and mutations in ribosomal proteins on the accuracy of the two-step selection of aminoacyl-tRNAs in protein biosynthesis. J. Biol. Chem. 256: 6676-6681.

Tranque, P., Hu, M.C., Edelman, G.M., and Mauro, V.P. 1998. rRNA complementarity within mRNAs: A possible basis for mRNAribosome interactions and translational control. Proc. Natl. Acad. Sci. 95: 12238-12243.

Weiss, R., Dunn, D., Dahlberg, A., Atkins, J., and Gesteland, R. 1988. Reading frame switch caused by base-pair formation between the $3^{\prime}$ end of $16 \mathrm{~S}$ rRNA and the mRNA during elongation of protein synthesis in Escherichia coli. EMBO J. 7: 1503-1507.

Westhof, E. and Fritsch, V. 2000. RNA folding: Beyond Watson-Crick pairs. Struct. Fold. Des. 8: R55-R65.

Wintermeyer, W., Peske, F., Beringer, M., Gromadski, K.B., Savelsbergh, A., and Rodnina, M.V. 2004. Mechanisms of elongation on the ribosome: Dynamics of a macromolecular machine. Biochem. Soc. Trans. 32: 733-737.

Yusupova, G.Z., Yusupov, M.M., Cate, J.H., and Noller, H.F. 2001. The path of messenger RNA through the ribosome. Cell 106: 233-241.

Yusupova, G., Jenner, L., Rees, B., Moras, D., and Yusupov, M. 2006. Structural basis for messenger RNA movement on the ribosome. Nature 444: 391-394.

Zhuang, Y. and Weiner, A.M. 1986. A compensatory base change in U1 snRNA suppresses a $5^{\prime}$ splice site mutation. Cell 46: 827-835. 

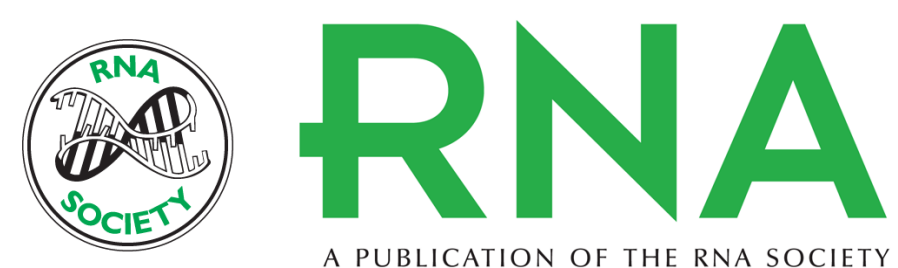

A PUBLICATION OF THE RNA SOCIETY

\section{Saturation mutagenesis of a +1 programmed frameshift-inducing mRNA sequence derived from a yeast retrotransposon}

Carla Guarraia, Laura Norris, Ana Raman, et al.

RNA 2007 13: 1940-1947 originally published online September 19, 2007

Access the most recent version at doi:10.1261/rna.735107

References This article cites 69 articles, 25 of which can be accessed free at: http://rnajournal.cshlp.org/content/13/11/1940.full.html\#ref-list-1

License

Email Alerting Receive free email alerts when new articles cite this article - sign up in the box at the Service top right corner of the article or click here. 